\title{
Discussion: A new look at rigid concrete pavement design
}

\section{B. P. Hughes}

Stuart Alexander, WSP Group, UK

I am investigating cracks in the floor slab of a single-level basement $215 \times 70 \mathrm{~m}$ used for car parking. The slab is $350 \mathrm{~mm}$ thick, and designed as a flat slab on pile caps at two-way centres between 5.5 and $6.5 \mathrm{~m}$. It was built nearly nine years ago, although the cracks were only observed about three years ago. They are roughly parallel, at right angles to the long dimension. They start about $27 \mathrm{~m}$ from each end and are in groups of three to six, spaced at between 500 and $1100 \mathrm{~mm}$, with a gap of $2 \mathrm{~m}$ or more before the next group. They are now around $0.5 \mathrm{~mm}$ wide, although filled with a dark grey precipitate having leaked extensively over the last three winters (apparently being below the water table for at least part of that time).

I believe the explanation is to be found in your paper, which in spite of its title is a very helpful commentary on BS $8007 .{ }^{3}$ It is that the cracks did initially form as predicted by BS 8007 with $\rho \geqslant \rho_{\text {crit }}$ (the critical steel ratio) but because $\rho \leqslant 1 \cdot 8 \rho_{\text {crit }}$ ( $0 \cdot 38 \%$ in the middle strip, $0 \cdot 60 \%$ in the column strip) they then widened uncontrollably rather than additional new cracks forming. This is predicted by your paper, but I have not found any other reference to it.

Presumably BS $8007^{3}$ is what its title says-that is, a code of practice for design of concrete structures to retain aqueous liquids. Most such structures are not only full of water but are backfilled with soil, and many have a roof also covered with soil. They are therefore protected from shrinkage and temperature drop, so that design for early thermal contraction is sufficient for subsequent behaviour also. Basements and highway slabs are not so protected and thus behave differently.

I have the following additional comments and queries on your paper.

(a) In section 2, lines 11-14, you say that BS 8007 'primarily considered the early thermal movement in the immature concrete, and assessed the probable maximum crack widths due to all the thermal and shrinkage movements [my emphasis] accordingly.' This implies that it does so correctly, whereas the mature concrete clauses (in Appendix B of BS8007) start with the proviso that the strain in the reinforcement is limited to $0 \cdot 8 \boldsymbol{f}_{\mathrm{y}} / E_{\mathrm{s}}$ (with no guidance on how to check this). They then quote the
BS 8110 formulae for crack widths in well-reinforced sections in bending.

(b) In section 3, I assume that the tensile strength given by equation (1) is intended to be the average, in which case is $\boldsymbol{f}_{\mathrm{cu}}$ the characteristic (i.e. minimum specified) cube strength-or should it be the average (usually taken as $10 \mathrm{~N} / \mathrm{mm}^{2}$ higher)?

(c) In section 4, second paragraph, why the 'widest' cracks, why do they become 'even wider', and what do you mean by 'fully developed'?

(d) Should there not be an allowance for shrinkage in equation (6)?

(e) Section 4, last paragraph. I believe this applies to my basement slab also. However, I am puzzled by 'flexural and thermal fatigue'. I prefer to think of it as sustained loading, in which case I understand that the tensile strength is reduced by about $0 \cdot 7$. Assuming some of the contraction is still from short-term temperature drop, perhaps $0 \cdot 8$ is better-that is, a limit of $1.45 \rho_{\text {crit }}$ rounded up to $1.5 \rho_{\text {crit }}$.

$(f)$ BS 8007 (clause A3) ${ }^{3}$ states that the ratio $f_{\mathrm{ct}} / f_{\mathrm{b}}$ (where $f_{\mathrm{ct}}$ is the tensile strength at three days of concrete and $f_{\mathrm{b}}$ is the average bond strength between concrete and steel) is 'appreciably lower for mature concrete'. Can you advise on how much lower, as I assume that the formulae for $s_{\max }$ (the predicted maximum probable spacing of cracks for fully developed early thermal cracking) and $w_{\max }$ (the maximum predicted crack width) can also be applied to mature concrete?

\section{Author's reply}

I thank Mr Alexander for his interest in the paper and for his very perceptive queries, which highlight several very important aspects for concrete pavements. Taking the general comment first then, water-retaining structures are clearly subjected in general to less long-term drying shrinkage as well as far less thermal fatigue and load reversal than pavements. However, the essential fact that has not been appreciated for pavement design is that the early thermal effects produce a pre-formed system of cracking that can completely dominate the subsequent structural behaviour and performance. Thus the conclusion that early thermal cracking in the immature concrete is not of major importance for pavements is quite wrong, and this serious misconception is unfortunately still held for most current design approaches for continually reinforced concrete pavements (CRCP). 
All Mr Alexander's queries, including the floor slab problem, have direct and important relevance to pavements and can also indicate how apparent anomalies in pavement behaviour in different countries or situations can be readily explained. My responses to the detailed queries, followed by the floor slab problem with its parallels in CRCP, are as follows.

(a) The short answer is that Appendices A and B in BS $8007^{3}$ are intended to complement each other, and each should be applied independently to ensure that excessive crack widths are avoided. However, this query also highlights the similarity between the misconception held previously for thin reinforced sections generally and that still held today for CRCP. Neither the existence of a pre-existing system of early thermal cracks in the mature concrete, nor the major differences between the cracking behaviours in immature and in mature concrete, can be ignored. Appendix A for predicting crack widths in immature concrete is essentially based on Vetter's classical assumption for bond slip, ${ }^{7}$ whereas Appendix B for flexure and direct tension in mature concrete assumes the exact opposite: that is, that no slipping occurs at the surface of the reinforcement. ${ }^{3,5}$ In 1970, CP $2007^{1}$ suffered from two major defects, as follows. (i) Concrete sections were theoretically designed so that tensile stresses due to external loading did not crack the concrete in either direct tension or bending. This made $\mathrm{RC}$ construction for water-retaining structures very uneconomic, and exacerbated the problems for early thermal cracking owing to the very thick sections that were required. (ii) The clauses for control of 'shrinkage' cracking were very unsatisfactory, not least because early thermal cracking was virtually ignored. Allowable joint spacings were too wide and minimum reinforcement ratios were too low, such that, even when used in combination, excessive cracking occurred. Despite early work at Birmingham ${ }^{8,9}$ and Technical Note $21,{ }^{10}$ which was one of CIRIA's bestsellers, conventional design for 'shrinkage' remained geared to thinking primarily in terms of mature concreteinstead of primarily to early thermal contraction in immature concrete and then to the thermal and long-term drying shrinkage in the mature concrete. Thus when drafting the clauses for BS $5337^{2}$ and to a lesser extent for BS 8007 the approach to controlling thermal and shrinkage contraction was still controversial. Indeed, the paper in Concrete (May 1972) ${ }^{11}$ was almost rejected because the industry at that time was not ready to accept a paper that emphasised that concrete will generally crack owing to early thermal effects and that this cracking should be suitably controlled. In contrast, the clauses that existed in BS $8110^{5}$ at that time for estimating crack widths in mature concrete due to external loading-either bending or direct tension-were generally accepted and appeared to give satisfactory predictions. Hence the main concern even in 1987 was for Appendix A. Appendix B in BS 8007 simply adapted and incorporated the appropriate expressions from BS 8110. Designing for a given maximum crack width in any direction as indicated in the paper is far superior to using vague guidelines for the average longitudinal crack spacing in the mature concrete for flexural and other effects that are superimposed on a preformed system of early thermal cracks.

(b) I agree that the cube strength to be inserted in equation (1) should ideally be the average cube strength and not the (minimum) characteristic value. However, the following practical considerations should be borne in mind. Whereas some $20 \%$ excessives above the design crack widths were considered acceptable for the actual crack widths, uncontrolled cracks were not acceptable as such cracks could become extremely wide. Thus the use of partial safety factors (hidden or otherwise) to ensure a very low probability of the steel yielding due to early thermal contraction was essential. Pavements and other slabs that are not on a sliding layer induce significant frictional forces, which oppose the direction of movement of the immature concrete as it contracts and thus assist the tensile force in the reinforcement across a crack as it widens. Furthermore, for compatibility of steel strains the steel stress at sections that have not cracked and are remote from the crack must be compressive. ${ }^{4,8,9}$ Neglecting the compressive force that develops at these adjacent sections provides in effect a further hidden safety factor. Again, although a normal Gaussian distribution of cube strengths can generally be assumed, actual distributions tend to be slightly skewed away from very high strengths. Taken together, these hidden safety factors can justify the use of the characteristic cube strength in equation (1) and thus simplify the procedure.

(c) The maximum cracking mechanism given in BS 8007 predicts the maximum number of cracks that could occur theoretically if the overall thermal and shrinkage contraction were very large ${ }^{4}$ (for detailed analysis see Reference 13). Fortunately in practice the overall contraction is very much less, and so only a few of these cracks develop full bond slipping over the predicted bond length (i.e. fully develop). Furthermore, it is only these 'live' cracks that will tend to become even wider with further contraction strain (e.g. long-term drying shrinkage), as predicted by equation (6).

(d) Yes. BS 8007 does in fact include terms for the estimated shrinkage strain and for the ultimate concrete tensile strain. However, for exposure of pavements to normal UK climatic conditions these terms can very often virtually cancel each other out, and the code suggests the use of the simplified equation (6). Clearly, where the long-term shrinkage strain is greater-that is, when protected from the weather or exposed to more arid conditions elsewhere in the worldthis simplification should not be used.

(e) Where the thermally cracked CRCP is essentially only oneway spanning with ground beam anchorage at either end, as in current conventional designs, $1 \cdot 8 \rho_{\text {crit }}$ is considered advisable. This is similar to the amount that has generally been provided, and is considered necessary in any case because the transverse reinforcement has usually been inadequate. However, the 'thermal shunt' concept ${ }^{12}$ indicates that a much lower limit of $1 \cdot 25 \rho_{\text {crit }}$ should be sufficient to ensure controlled movement in the longitudinal direction between individual cracked segments. This clearly assumes a two-way spanning pavement suitably designed for narrow maximum design crack widths in both directions, as indicated in the paper. For typical one-way spanning designs not specifically focused to ensure an adequately fine maximum crack width even for the longitudinal reinforcement, then the fatigue life of pavements with less than $1.5 \rho_{\text {crit }}$ is likely to 
be very short. A given $w_{\max }$ in equation (6) clearly becomes more difficult to achieve as the steel ratio is reduced. The basement floor slab and the thermal shunt concept are again considered later. Regarding flexural and thermal fatigue this occurs as a result of widely fluctuating and frequent reversals of stress and is more severe than a fairly constant sustained load.

$(f)$ The formula for $s_{\max }$ assumes bond slipping, whereas that for direct tension in Appendix B assumes no slip. However, some bond slipping clearly does occur in mature concrete, and this is in fact assumed in equation (6). The restraint factor for $\alpha T_{2}$ (and for a long-term drying term) is taken in BS 8007 as being the same as that for $\alpha T_{1}$ but for different reasons. The bond creep for immature concrete is extremely high: hence $R$ is taken as 0.5 effectively for full external restraint for the bond length $s_{\min }$ (equals $s_{\max } / 2$ ). Bond slipping for a given tension takes place over shorter bar lengths as the bond strength increases with age. Thus the bond creep for mature concrete is much less, but so is the effective bond length: hence the product $R s_{\max }$ can be assumed for simplicity to be the same as for immature concrete. ${ }^{4}$ Similarly the formulae for $s_{\max }$ and $w_{\max }$ can include terms for long-term drying shrinkage as necessary. For a mature member subjected to a direct tensile load then some additional bond slipping must clearly take place, but this will be less than if bond slipping due to thermal stresses had already taken place in the immature concrete followed by further movement under thermal cycling.

The car parking floor slab problem is complicated by a number of factors. If the mature floor slab were subjected to a particularly low ambient temperature at some time in its history it would be similar to the common case of CRCP cast directly on the sub-base and with ground beam anchorage at either end. Your assumption that the steel had yielded in tension could then be correct. However, it is much more likely that the wide cracks occurred in the immature concrete. In this case it would be similar to the case of CRCP cast on a lowfriction polythene membrane. This practice has been used for CRCP in Australia, and has resulted in much wider early thermal cracks being observed. When drafting the appropriate clauses for BS 8007 only the most relevant aspects for waterretaining structures (or pavements) were included. The essential concern at that time was to emphasise the major differences in the basic assumptions that were appropriate for immature and mature concrete respectively. The outstandingly successful application of BS 8007 subsequently, together with my consulting experience resolving excessive cracking problems and disputes, has indicated that the inclusion of some other aspects could be of considerable benefit, but a suitable opportunity for a later revision did not occur. Three important aspects that are relevant both to pavements and to the floor slab that are not covered in the paper are as follows.

(a) Upper bound solution for early thermal cracking in immature concrete. A comparison of the various formulae that have been proposed for early thermal cracking of concrete has been presented elsewhere. ${ }^{13}$ The maximum cracking mechanism given in Appendix A of BS 8007 predicts the maximum number of cracks that can occur theoretically and applies to the usual situation where significant side restraint is present between the end restraints-for example CRCP with friction between pavement and sub-base. However, a suspended slab with full end restraint and very low friction (or CRCP with a continuous smooth polythene membrane) involves a minimum cracking mechanism. This mechanism predicts the minimum number of cracks (and hence the maximum individual crack width) that can occur theoretically for a given applied strain. Notice that for this mechanism considerable strain energy is released at each crack when it occurs as the temperature in the immature concrete falls back from the hydration peak. Consideration of the steel strain at a cracked section when the concrete is about to crack at a section leads to the upper bound expression given by Bricknell and Hoadley in 1976: ${ }^{14}$

9

$$
w_{\lim }=\boldsymbol{\varepsilon}_{\mathrm{ult}} s_{\min } \frac{1+\alpha_{\mathrm{e}} \rho_{\mathrm{c}}}{\alpha_{\mathrm{e}} \rho_{\mathrm{c}}}
$$

where $\alpha_{\mathrm{e}}$ is the modular ratio, $\rho_{\mathrm{c}}$ is the steel ratio, and $\boldsymbol{\varepsilon}_{\mathrm{ult}}$ is the ultimate tensile strain of the immature concrete. Actual crack widths in practice will tend to be even higher than the above predicted value, and quite variable, as such cracks occur suddenly with shock as the stored strain energy is released. Observed crack widths can be 50\% or more greater than the above value. Again, the crack widths for a minimum cracking mechanism oscillate between the upper bound given by Bricknell and Hoadley and various lower bound values as further cracks occur with increasing restrained strains in the concrete. ${ }^{15}$ However, even the lower bound values for crack widths for a minimum cracking mechanism are wider than $w_{\max }$ for a maximum cracking mechanism, at all practical values of restrained strain, $\boldsymbol{\varepsilon}_{\mathrm{r}}$. Another major difference between the minimum and maximum cracking mechanisms is that whereas $w_{\max }$ increases directly with increasing $\boldsymbol{\varepsilon}_{\mathrm{r}}, w_{\text {lim }}$ is independent of $\boldsymbol{\varepsilon}_{\mathrm{r}}$. Thus the threshold width of a newly formed crack for a minimum cracking mechanism is large and increases relatively little to reach $w_{\text {lim }}$, whereas the corresponding threshold value for a maximum cracking mechanism is relatively small but continues to increase directly with increasing values of $\boldsymbol{\varepsilon}_{\mathrm{r}}$.

(b) Thick sections and temperature gradient effects. When the top surface of a pavement is exposed or the shuttering is removed, rapid cooling of the suddenly exposed immature concrete produces a temperature gradient between the surface and the core so that the reinforcement need only control cracking in the 'surface zone'. Once initiated, the crack can propagate subsequently into the core in order to control cracking in a thick section. This approach is already included in BS 8007, and the surface zone depths were essentially fairly arbitrary estimates based on experience with thick sections. However, they have continued to provide a very convenient and economical design approach that has proved to work well in practice. For surface zones in slabs under $300 \mathrm{~mm}$ thick (upper face only in pavements and ground slabs and both faces in wall slabs), surface zone depths can be taken as the lesser of twice the reinforcement depth or half the total slab thickness. Also, two further important observations can be made.

(i) Large temperature differences between exposed face 
and core can introduce cracks at relatively close spacing without any need for distribution reinforcement. The results of a finite-element analysis showing the predicted crack spacing for the surface of a plain concrete section $600 \mathrm{~mm}$ thick for various coreto-face temperature differences are given elsewhere. ${ }^{13}$ In warmer climates than that for the UK, crack spacing and crack widths have been found to be shorter and narrower than expected from BS 8007 for the fall from the early thermal hydration peak temperature. This is undoubtedly possible for large temperature differences between surface and core even for 'thin' sections as indicated above. However, to design for thermal shock at the surface is neither recommended nor considered feasible. The reinforcement in the surface zone should therefore in general be designed to BS 8007.

(ii) The surface zones as recommended in BS 8007 can also be applied to immature concrete members subjected to a minimum as well as a maximum cracking mechanism. ${ }^{16,17}$

(c) Full or partially controlled thermal movement for mature concrete. Section 4 of the paper shows that $1 \cdot 8 \rho_{\text {crit }}$ can give full control for thermal movement. However, where CRCP is used either with end anchorage or (preferably) universal beam anchorage incorporating expansion joints at each end then the thermal shunt concept can be taken into account. Significant savings in longitudinal reinforcement can be made, as adequate partial control for thermal movement should be possible with about $1 \cdot 25 \rho_{\text {crit }}$, especially where satisfactory two-way spanning for the cracked pavement is achieved as for the uncracked pavement. ${ }^{12}$ Utilisation of the thermal shunt concept can therefore effect significant economies in the amount of longitudinal reinforcement required, as a longitudinal steel ratio of as low as $0 \cdot 5 \%$ could be possible with careful design. It takes advantage of the fact that daily thermal cycles minimise the stress variations that occur in the reinforcement crossing 'live' thermal cracks in pavements throughout the major seasonal cycles from the hottest summer day to the coldest winter night.

Thus your car parking basement slab is seen to involve a 'thick' section with end restraint and little or no side restraint between intermediate partial restraints at pile caps from 5.5 to $6.5 \mathrm{~m}$ apart. The free movement at the ends indicates that four or more pile caps were required to build up effective full restraint in the central length. The top surface zone would have cooled first and cracked owing to the restraint exerted by the relatively hot central 'core'. These cracks would be according to the maximum cracking mechanism. They would then start to progress into the core in the normal way. However, the normal steady progression in this case would have been terminated quite suddenly as one crack reached some critical point. Any one of the surface zone cracks could trigger the sudden minimum cracking mechanism in the core. This crack could then become very wide (i.e. $w_{\text {lim }}$ )-and very much wider than the fully developed crack of the maximum cracking mechanism $\left(w_{\max }\right)$. One of these very wide cracks could possibly have occurred between each pile cap, giving the impression of uncontrolled cracking due to yielding of the reinforcement. Although the steel ratio within the surface zone should in general be more than $1 \cdot 1 \rho_{\text {crit }}$ to cover all eventualities (e.g. the surface zone taking longer than three days to cool down to ambient), it is unlikely that uncontrolled cracking within the relevant surface zones actually occurred in this case. It is far more likely that the very wide crack widths can be predicted by equation (9), with an allowance for shock loading.

Notice that the surface zone concept provides an invaluable design guide for achieving major economies in the reinforcement in thick pavements such as airport runways. Notice also that this concept works for thick sections subjected to minimum cracking mechanism conditions only, because it works for the maximum cracking mechanism that occurs first in the surface zone. For a variety of reasons, however, not least the shock that occurs at the time of cracking, the surface zone subsequently subjected to a minimum cracking mechanism should never be less than $1 \cdot 8 \rho_{\text {crit. }}$. Again, the 'thermal shunt' concept ${ }^{12}$ necessarily requires that the steel ratio for the full depth of the CRCP be not less than $1 \cdot 25 \rho_{\text {crit }}$ located at or below mid-height.

\section{REFERENCES}

7. VeTTER C. P. Strain in reinforced concrete due to volume changes. Transactions of the American Society of Civil Engineers, 1933, 98, 1039-1053.

8. Evans E. P. and Hughes B. P. Shrinkage and thermal cracking in a reinforced concrete retaining wall. Proceedings of the Institution of Civil Engineers, 1968, 39, 111-125; Discussion 40, 539-568.

9. Hughes B. P. and Miller M. M. Thermal cracking and movement in reinforced concrete walls. Proceedings of the Institution of Civil Engineers, 1970, 45, Supplement (iii), 65-86; Discussion Supplement (xix), 443-451.

10. Hughes B. P. Control of Thermal and Shrinkage Cracking in Restrained Reinforced Concrete Walls. Construction Industry Research and Information Association, London, 1971, CIRIA Technical Note 21.

11. Hughes B. P. Controlling shrinkage and thermal cracking. Concrete, 1972, 6, No. 5, 34-42.

12. Hughes B. P. A new look at bonded concrete overlays for pavements. Advanced Trends in Pavement Rehabilitation Design and Preservation, Proceedings of the $3 r d$ International Symposium on Maintenance and Rehabilitation of Pavements and Technological Control, MAIREPAV '03, Guimarães, Portugal, 2003, 585-593.

13. Hughes B. P. and MAHMood A. T. An investigation of early thermal cracking in concrete and the recommendations in BS 8007. The Structural Engineer, 1988, 66, No. 4, 61-69.

14. BRICKELl A. and HoADLEY P. J. Thermal and shrinkage cracking in reinforced concrete slabs. Civil Engineering Transactions (Barton), 1976, CE18, No. 1, 24-28.

15. Hughes B. P. and Videla Cifuentes C. Comparison of earlyage crack width formulas for reinforced concrete. ACI Structural Journal, 1988, 85, No. 2, 158-166; Discussion, 1989, 86, 105- 106.

16. Hughes B. P. and Mahmood A. T. Early thermal cracking in end-restrained thick reinforced concrete members. Proceedings of the Institution of Civil Engineers, Part 2, 1988, 85, 305-315; Discussion, 1989, 87, 155- 158.

17. Hughes B. P. Early thermal cracking in reinforced concrete. Proceedings of a European Conference on Cracking of Concrete and Durability of Construction, AFREM, SaintRemey-les-Chevreuse (Paris), 1988, pp. 2·35-2•42. 